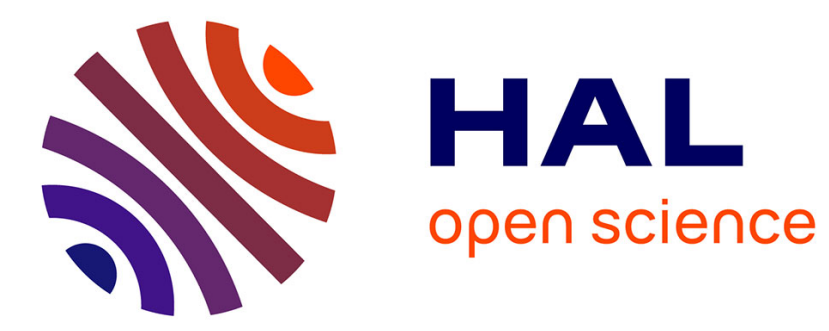

\title{
Gyroresonant Surfing Acceleration
}

Yasuhiro Kuramitsu, Vladimir Krasnoselskikh

\section{To cite this version:}

Yasuhiro Kuramitsu, Vladimir Krasnoselskikh. Gyroresonant Surfing Acceleration. Physical Review Letters, 2005, 94 (3), 10.1103/PhysRevLett.94.031102 . insu-03342820

\section{HAL Id: insu-03342820 \\ https://hal-insu.archives-ouvertes.fr/insu-03342820}

Submitted on 13 Sep 2021

HAL is a multi-disciplinary open access archive for the deposit and dissemination of scientific research documents, whether they are published or not. The documents may come from teaching and research institutions in France or abroad, or from public or private research centers.
L'archive ouverte pluridisciplinaire HAL, est destinée au dépôt et à la diffusion de documents scientifiques de niveau recherche, publiés ou non, émanant des établissements d'enseignement et de recherche français ou étrangers, des laboratoires publics ou privés. 


\title{
Gyroresonant Surfing Acceleration
}

\author{
Yasuhiro Kuramitsu* and Vladimir Krasnoselskikh \\ Laboratoire Physique et Chimie de l'Environnement, CNRS, 45071 Orléans, France
}

(Received 25 February 2004; published 25 January 2005)

\begin{abstract}
We discuss a new acceleration or energization mechanism of charged particles in space and astrophysical plasmas. In the presence of an electrostatic potential gradient and a circularly polarized electromagnetic monochromatic wave, particles are accelerated efficiently by keeping cyclotron resonance with the wave due to the electrostatic dragging force. In addition, particles can propagate against the electrostatic potential even if they have smaller parallel energy. This mechanism is potentially widely applicable, in terms of particle acceleration and transport, to various space and astrophysical phenomena, such as shock environment and short-large amplitude magnetic structures. We introduce the basic physical process of the acceleration or energization mechanism theoretically and numerically.
\end{abstract}

DOI: 10.1103/PhysRevLett.94.031102

Diffusive shock acceleration (DSA) is widely accepted as a major cosmic ray acceleration mechanism in astrophysical and solar system plasmas [1]. It is essentially the first order Fermi acceleration, and predictions of DSA agree well with the observed cosmic ray spectra. According to the angle between the upstream magnetic field and the shock normal direction $\theta_{B}$, the shock geometry falls into two classes, quasiparallel $\left(\theta_{B}<45^{\circ}\right)$ and quasiperpendicular $\left(\theta_{B}>45^{\circ}\right)$. The DSA is associated mainly with quasiparallel shocks, while shock drift acceleration plays a main role of particle acceleration at the quasiperpendicular shocks [2]. Plentiful nonlinear wave phenomena are observed at upstream of the Earth's bow shock [3], a relatively small and weak nonrelativistic version of the astrophysical shocks. Especially quasiparallel shocks show rather complicated wave activities. Direct in situ measurements give clear evidence that the quasiparallel shock can be presented as a patchwork of short-large amplitude magnetic structures (SLAMS) rather than a continuous transition [4]. An important opened issue is related to the injection mechanism of cosmic rays. To be efficiently accelerated, particles should have a large initial energy and be well separated from the thermal background; that is, so-called "seed" population should exist. Although the formation of this population has been discussed extensively $[5,6]$, in the context of ion acceleration at quasiparallel shocks, the electrostatic potential of shock is quite often ignored since the potential is believed to decelerate ions, and also because of the difficulties of observation [7]. Recent Cluster satellites carried out the first direct measurements of the electric field of SLAMS at a quasiparallel shock [8]. On the other hand, in situ studies of particle distribution functions allowed one to identify several ion populations and their relation to different forms of the wave activity at the quasiparallel shock front [9]. Diffuse ions with broader distribution in energy and pitch angle are accompanied by large amplitude, low-frequency, wideband spectral waves. Before this diffusive stage, intermediate or so-called "gyrating" ions with narrower distribution are observed associated with low-frequency quasimonochro-
PACS numbers: 96.50.Pw, 52.20.Dq, 52.25.Fi, 96.40.-z

matic waves. Since the direct in situ observations of particles and related wave activities are impossible in astrophysical plasmas, a key to understanding the injection problem and further acceleration of cosmic rays is to reveal the relation between the observed populations and shock structure including the wave activities by using in situ observations.

In this Letter, we focus on the ion motion at shock-type structures accompanied by an electromagnetic circularly polarized wave. To model an effect of such a shock front on particle motion, we present it as an electrostatic potential profile. We propose an acceleration or energization mechanism due to a combined action of electrostatic potential and circularly polarized electromagnetic wave. We describe its major features theoretically and numerically.

Let us consider a monochromatic electromagnetic wave propagating parallel to a background magnetic field and also to an electrostatic potential profile varying along the same direction. The basic equations are the equations of ion motion,

$$
m \frac{d \boldsymbol{v}}{d t}=e\left(\boldsymbol{E}+\frac{\boldsymbol{v}}{c} \times \boldsymbol{B}\right)
$$

with the electric and magnetic fields given by $\boldsymbol{E}=$ $\left(-\partial \varphi / \partial x, E_{y}, E_{z}\right)$ and $\boldsymbol{B}=\left(B_{0}, B_{y}, B_{z}\right)$, where $B_{0}$ is a magnitude of background magnetic field. Here we do not take into account wave amplitude and wavelength change while crossing the shock front or SLAMS. The electromagnetic wave is written as follows:

$$
\tilde{b}=b_{y}+i b_{z}=b \exp \left[i\left(k x-\omega t+\phi_{w}\right)\right],
$$

where $b$ is the wave amplitude normalized to the background magnetic field, $k$ is the wave number, $\omega$ is the wave angular frequency, and $\phi_{w}$ is the phase constant. The transverse electric field is determined from $\boldsymbol{k} \times \boldsymbol{E}=$ $\omega \boldsymbol{B} / c$. Using complex variable $\tilde{\boldsymbol{v}}=\boldsymbol{v}_{y}+i v_{z}$, we write the perpendicular component of Eq. (1) as 


$$
\begin{aligned}
\frac{d}{d t}[\tilde{\boldsymbol{v}} \exp (i \Omega t)]= & -i \Omega\left(c_{p}-v_{x}\right) b \\
& \times \exp \left[i\left(k x-\omega t+\Omega t+\phi_{w}\right)\right],
\end{aligned}
$$

where $c_{p} \equiv \omega / k$ is the wave phase velocity, and $\Omega$ is ion gyrofrequency. We focus on the resonant particles satisfying the condition $\omega-k v_{x}-\Omega=0$. These particles in the presence of a finite amplitude wave are trapped; thus, the parallel velocity in the absence of any other forces oscillates around the resonant velocity. If one could force particles to keep the exact resonant condition, such a wave particle interaction will lead to a particle acceleration. To show this, let us assume that $v_{x}=v_{r} \equiv$ $(\omega-\Omega) / k$, and thus $x=x_{0}+v_{r} t$. Integrating Eq. (3) gives

$$
\tilde{v}=-i \frac{\Omega^{2} b}{k} t \exp \left[i\left(\phi_{w}-\Omega t\right)\right]+v_{\perp 0} \exp \left[i\left(\phi_{p}-\Omega t\right)\right],
$$

where $v_{\perp 0}$ and $\phi_{p}$ are determined by initial conditions, and we set $x_{0}=0$. If we assume that this "forced resonance " is kept by supplying an electrostatic potential, it can be found to have the form

$$
\frac{e \varphi}{m}=\frac{\Omega^{3} b^{2}}{2 k v_{r}} x^{2}+\Omega b v_{\perp 0} x \sin \psi_{0},
$$

where $\psi_{0}=\phi_{w}-\phi_{p}$ is the initial phase difference between the wave and the particle and time is related with space as $x=v_{r} t$. The first and second terms of the righthand side of Eq. (4) give oscillation with growing amplitude and Larmor motion, respectively. We can write the first term of $v_{y}$ as $\Omega^{2} b t \sin \left(\theta_{w}-\Omega t\right) / k$ and $E_{y}$ that the particle will see as $c_{p} B_{w} \sin \left(\theta_{w}-\Omega t\right) / c$. Thus, in the presence of such an external force, particles can be accelerated monotonically in a perpendicular direction by the wave electric field, similar to the surfing acceleration $[6,10]$. In the conventional mechanism particles are accelerated by a tangential component of the electric field induced by the motion of the front perpendicular to the magnetic field. In our case particles are accelerated by wave electric field, rotating in phase with the electric field of the electromagnetic wave. We refer to this acceleration or energization mechanism as a gyroresonant surfing acceleration. We start from illustrating that not only particles in exact resonance can gain energy but also those close to it with the potential Eq. (5); then we extend the results to general form of potential at parallel shocks.

We show in Fig. 1 trajectories of a resonant (solid lines) and a near-resonant (dashed lines) ion in $x-v_{x}$ space together with the electrostatic potential (dotted line) 1(a), in perpendicular velocity space 1(b), and time evolution of $v_{y}$ and $E_{y} 1(\mathrm{c})$ obtained by numerical integration of Eq. (1), where the electrostatic potential is given by Eq. (5). We normalized all the variables using $B_{0},\left|c_{p}\right|$, and $\Omega$ evaluated at $B_{0}$. From now on we omit these con-
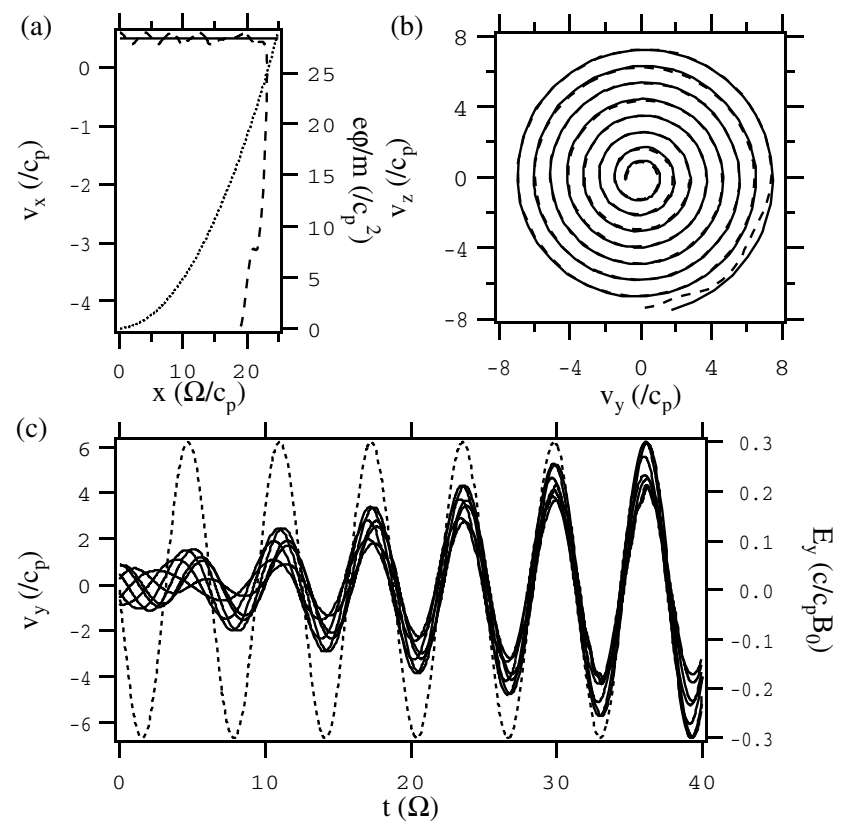

FIG. 1. Resonant (solid lines) and near-resonant (dashed lines) ion trajectories are shown in $x-v_{x}$ space with electrostatic potential (dotted line) (a), and in perpendicular plane (b). Time evolution of $v_{y}$ (solid lines) and $E_{y}$ (dotted line) are shown in (c).

stants. Parameters are $b=0.3, k=2, c_{p}=1, \psi_{0}=3$ [in 1 (c) $\psi_{0}$ ranges $\left.[0,2 \pi]\right], v=1, v_{x}=0.5$ for the resonant ion, and $v_{x}=0.6$ for the near-resonant ion. In Fig. 1(a), the resonant ion propagates against the electrostatic potential (dotted line) keeping the parallel velocity constant. From Fig. 1(b), the ion accelerates monotonically in the perpendicular direction while satisfying the cyclotron resonance condition along the trajectory. On the other hand, for the near-resonant ion, although the derivation of Eq. (5) is not exact, we still see similar trajectories; the ion is accelerated quite efficiently while propagating against the electrostatic potential and keeping the parallel velocity approximately constant. In Fig. 1(c), we can see the phase synchronization as we described above. Ions become oscillating in phase with electromagnetic electric field as time has passed resulting in efficient energy gain.

The acceleration of near-resonant ions suggests that gyro-resonant surfing can take place in the presence of a wider class of potential fields. Growing electric potential fields, such as dotted lines in Fig. 1(a), are common in the vicinity of quasiparallel shocks [11], as well as circularly polarized electromagnetic waves [12]. Thus we assume a model electric field of the form as

$$
E_{x}=-a \operatorname{sech}^{2}\left(\frac{x}{l}\right)
$$

which gives a tanh-type electrostatic potential with a potential difference $\Delta \varphi=2 a l$. Let us define a reference system with the profile at rest as the $S$ system and a upstream plasma frame as the $P$ system. We choose the 
system such that both the wave and the ions are convected to the profile by the plasma flow $v_{u}$. To study characteristics of the acceleration, we consider a shell distribution with a thermal velocity $v_{t}$ as a part of upstream plasma. We inject the ions from far upstream of the profile.

At first we choose the parameters so that the initial shell includes the resonance velocity in order to show the basic properties of gyroresonant surfing. Parameters are $b=0.2$, $c_{p}=-1, k=0.4, v_{r}=-3.5, v_{t}=4$ in the $P$ system, and $v_{u}=4$ in the $S$ system. Figure 2(a) shows the initial velocity distribution of the ions (blue crosses) and the distribution after interaction (red dots) in the $S$ system. When the electromagnetic wave is absent, i.e., $b=0$, particle reflection is determined by the parallel energy; ions with $m v_{x}^{2} / 2>\Delta e \varphi$ go through the potential, otherwise are reflected "specularly," $v_{x} \rightarrow-v_{x}$ [green circles in Fig. 2(a)]. When the electromagnetic wave exists, we can see an efficient perpendicular acceleration around the resonant velocity (dotted line). Figures 2(b) and 2(c) show trajectories of a resonant ion $\left(v_{x}=v_{r}=0.5\right.$ in the $S$ system) from Fig. 2(a). The initial phase difference is chosen to $\psi_{0}=k x_{0}+\phi_{w}-\phi_{p}=3$. In Fig. 2(b) $(x-$ $v_{\perp}$ space), the ion propagates against the potential even though the parallel energy is much smaller than the potential, and is accelerated efficiently at the profile. Figure 2(c) shows ions trajectory in the velocity space, where $S, W, P$,
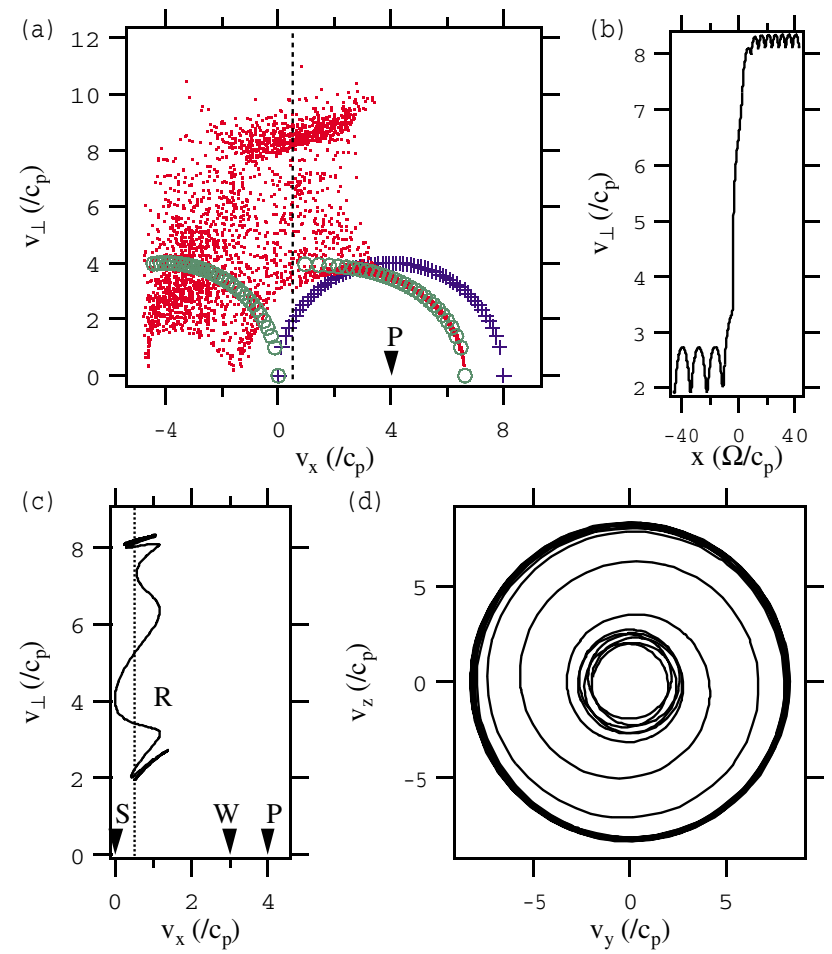

(d)

(d)

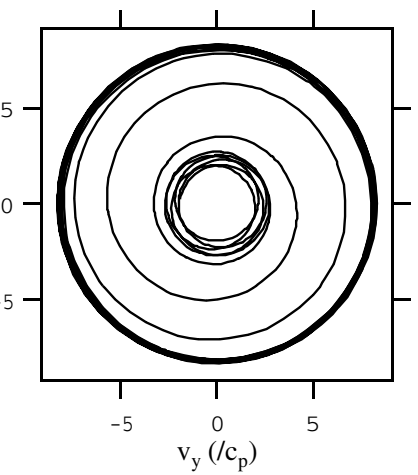

FIG. 2 (color). Blue crosses and red dots (green circles) represent the ions' velocity distribution before and after interaction with the model electric field in the presence (absence) of the electromagnetic wave (a). Resonant ion trajectories are shown in $x-v_{\perp}$ space (b), in velocity space (c), and in perpendicular velocity space $(\mathrm{d})$. and $R$ represent the $S$ system, the wave phase velocity, the $P$ system, and the resonant velocity, respectively. The ion is accelerated in the perpendicular direction, keeping the parallel velocity around the resonant velocity (dotted line). In Fig. 2(d) (perpendicular velocity space), one can see a trajectory similar to those in Fig. 1(b), although the potential shape is considerably different. Figure 3(a) shows the dependence of perpendicular acceleration of ions in Fig. 2 on the initial pitch angle and phase. Here $\mu$ is the pitch angle cosine in the $P$ system. The color scale represents perpendicular velocity of ions after interaction with the profile. Figure 3(b) shows ion trajectories upstream of the profile in the same phase space. Ions are trapped around $\mu \sim v_{r} / v$ and $\psi=\pi$ in the phase space. There is clear evidence that gyroresonant surfing acceleration is especially efficient for trapped particles.

We now apply the mechanism to Earth's bow shock, choosing parameters appropriate to ion beam-excited Alfvén waves $\left(k=0.1, c_{p}=-1, v_{u}=5.5, v_{t}=1, b=\right.$ $\left.0.5,2 a l=m v_{u}^{2} / 2, k l=1\right)[4,8,11-13]$. Figure 4 shows distributions of the particles before and after interaction as the same as Fig. 2(a). There are distinct two components: one is thermal, low energy particles, and the other is nonthermal, high energy particles. Some of the thermal particles have parallel velocity less than zero; however, this is simply due to oscillations in the large amplitude wave. All the thermal particles are downstream particles and all the nonthermal particles are reflected upstream ones. Characteristic energy of nonthermal particles is $\sim 5$ times the energy of upstream flow, which is consistent with the
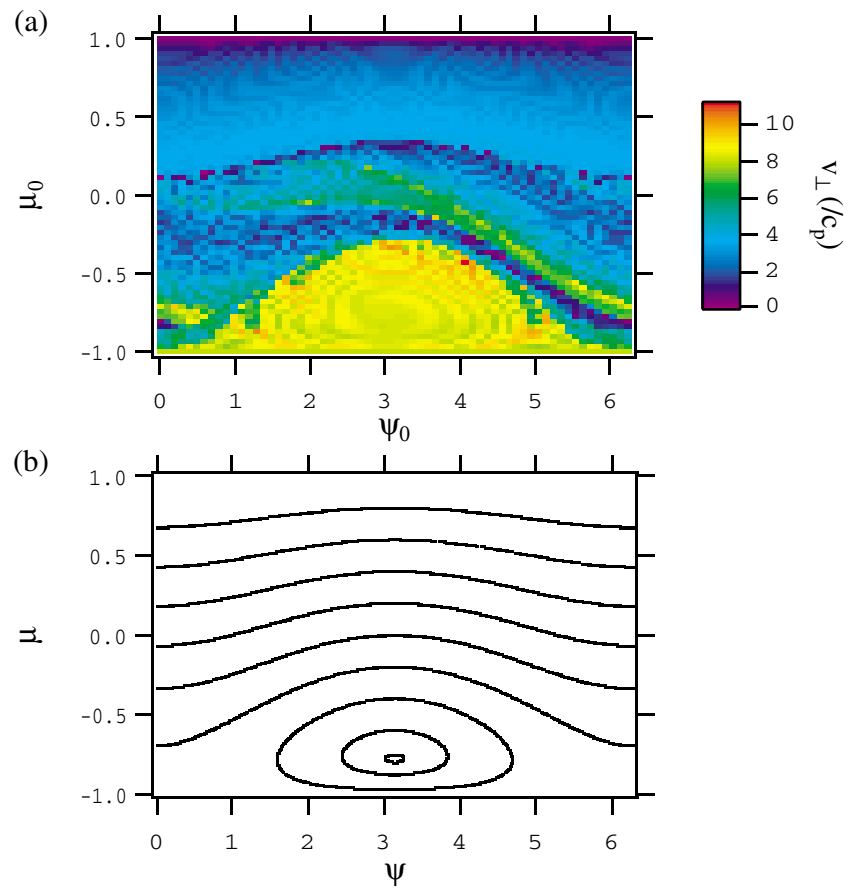

FIG. 3 (color). Initial pitch angle and phase dependence of gyroresonant surfing acceleration is shown (a). Trajectories of upstream ions in the same phase space are shown (b). 


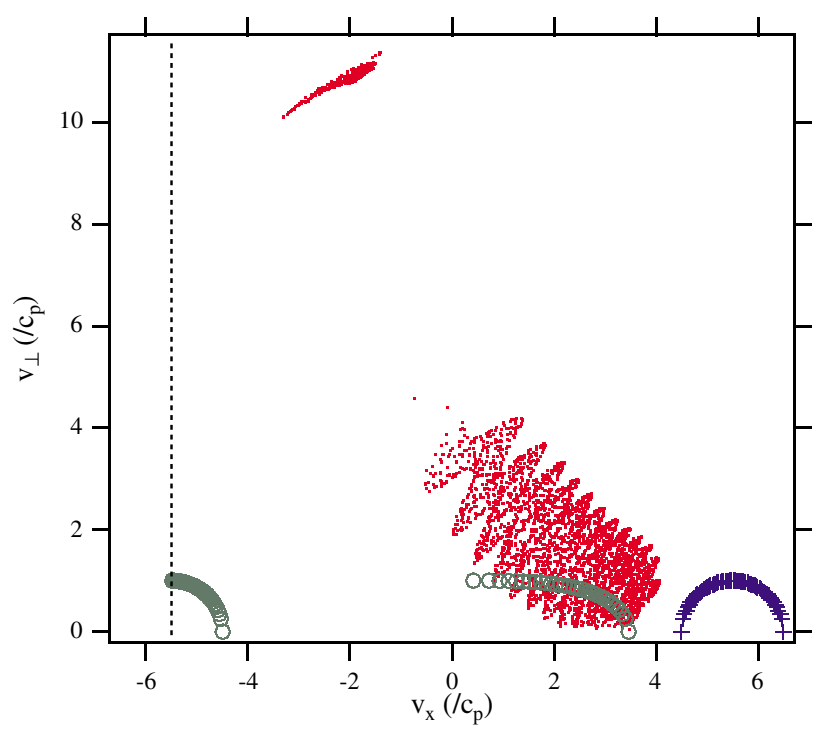

FIG. 4 (color). The same as Fig. 2(a), particle distributions are shown using realistic parameters.

typical energy of observed intermediate ions [9]. Note that all the particles do not satisfy the resonance condition, where $v_{r}=-5.5(-11)$ in the $S(P)$ system. Thus, ions are not trapped by the wave initially. Interacting with the potential, the reflected nonthermal particles are trapped before and after reflection, resulting in efficient acceleration.

We have discussed a new acceleration or energization mechanism due to the combination of two effects: the trapping of particles by the circularly polarized electromagnetic wave and the dragging by the electrostatic electric field to keep the resonance condition. This results in the efficient perpendicular acceleration of particles, and allows them to propagate downstream even when they have smaller kinetic energy than the potential field. This can be understood as follows. From the perpendicular component of Eq. (1) we calculate the time derivative of perpendicular particle energy,

$$
\frac{d}{d t}\left(\frac{1}{2} v_{\perp}^{2}\right)=\Omega\left(c_{p}-v_{x}\right)\left(v_{y} b_{z}-v_{z} b_{y}\right) .
$$

When $d v_{x} / d t \sim 0$, we can replace $\Omega\left(v_{y} b_{z}-v_{z} b_{y}\right)$ by $(e / m) \partial \varphi / \partial x$. Furthermore, if the parallel velocity of particles is close to the resonant velocity, $\left(c_{p}-v_{x}\right)$ in Eq. (7) can be written as $\Omega / k$, which is considered to be positive. Under such conditions particles are accelerated in the perpendicular direction. Moreover, in the presence of elliptically, linearly polarized waves, and turbulence (described by the superposition of monochromatic waves) the gyroresonant surfing can take place. A similar mechanism can also potentially accelerate electrons using the whistler precursor wave. Furthermore, the gyroresonant surfing can operate in the same way if the dragging force is provided by a magnetic mirror. It is worth noting that the same mechanism can accelerate pickup ions in a cometary or solar wind plasmas [6].

The gyroresonant surfing can provide an efficient injection mechanism. The particles from the thermal background can be energized to form a seed particle population that can be further accelerated by DSA. This Letter provides the essential idea of gyroresonant surfing acceleration. The full theory of the formation of the seed particle population should include several additional effects, such as the inhomogeneity of the ambient magnetic field and/or oblique wave propagation. To develop the theory for astrophysical applications, it is necessary to include relativistic effects.

The authors thank B. Lefebvre, V. Lobzin, T. Hada, H. Kucharek, and M. Scholer for their helpful comments. This work was supported by the STUDIUM program.

*Electronic address: ykuramit@cnrs-orleans.fr

[1] G. F. Krimskii, Dokl. Akad. Nauk SSSR 234, 1306 (1977); W. I. Axford, E. Leer, and G. Skadron, in Proceedings of the 15th International Conference on Cosmic Rays (B'lgarska Akademiia na Naukite, Sofia, 1978), Vol. 11, p. 132; A. R. Bell, Mon. Not. R. Astron. Soc. 182, 147 (1978); R. D. Blandford and J. P. Ostriker, Astrophys. J. 221, L29 (1978).

[2] For a review, see T. P. Armstrong, M.E. Press, and R. B. Decker, Collisionless Shocks in the Heliosphere: Reviews of Current Research, edited by B. T. Tsurutani and R. G. Stone (AGU, Washington, DC, 1985), p. 271.

[3] For a review, see W. P. Wilkinson, Planet. Space Sci. 51, 629 (2003).

[4] S. J. Schwartz and D. Burgess, Geophys. Res. Lett. 18, 373 (1991); S. J. Schwartz et al., J. Geophys. Res. 97, 4209 (1992).

[5] For reviews, see M. Scholer, H. Kucharek, and K. J. Trattner, Adv. Space Res. 21, 533 (1998); F. C. Jones and D. C. Ellison, Space Sci. Rev. 58, 259 (1991).

[6] G. P. Zank et al., J. Geophys. Res. 101, 457 (1996); M. A. Lee, J. Geophys. Res. 101, 4777 (1996); G. P. Zank et al., Phys. Plasmas 8, 4560 (2001).

[7] A. J. Hull et al., J. Geophys. Res. 105, 20957 (2000); Z. Kuncic, I. H. Cairns, and S. Knock, J. Geophys. Res. 107, SSH 11 (2002).

[8] R. Behlke et al., Geophys. Res. Lett. 30, 26 (2003).

[9] M. M. Hoppe et al., J. Geophys. Res. 86, 4471 (1981).

[10] R.Z. Sagdeev, Reviews of Plasma Physics, Vol. 4, edited by M. A. Leontovich (Consultants Bureau, New York, 1966), p. 23; R.Z. Sagdeev and V.D. Shapiro, JETP Lett. 17, 279 (1973); T. Katsouleas and J. M. Dawson, Phys. Rev. Lett. 51, 846 (1983).

[11] M. Scholer, H. Kucharek, and I. Shinohara, J. Geophys. Res. 108, SSH 3 (2003).

[12] M. M. Hoppe and C. T. Russell, J. Geophys. Res. 88, 2021 (1983).

[13] M. Hoshino and T. Terasawa, J. Geophys. Res. 90, 57 (1985). 